\title{
Numbers in radiology and personalized medicine: A commentary
}

\author{
Abdulwahab Alahmari \\ Radiology Specialist, Ministry of Health, Abha, Kingdom of Saudi Arabia.
}

Corresponding Author: Abdulwahab Alahmari, Radiology Specialist, Ministry of Health, Abha, Kingdom of Saudi Arabia.

\section{Received Date: 16 August 2021 | Accepted Date: 04 September 2021 | Published Date: 13 September 2021}

Citation: A Alahmari. (2021). Numbers in radiology and personalized medicine: A commentary. International Journal of Clinical Case Reports and Reviews. 8(3); DOI:10.31579/2690-4861/167

Copyright: (C) 2021 Abdulwahab Alahmari, This is an open-access article distributed under the terms of the Creative Commons Attribution License, which permits unrestricted use, distribution, and reproduction in any medium, provided the original author and source are credited.

\begin{abstract}
Some of the numbers in radiology used as a criteria to make or role out a diagnosis for some conditions. These numbers should not be taken for granted since personalized medicine state that every patient is different and should be treated based on his/her condition. Those numbers can't be used as a golden slandered in all case. This commentary will focus on some examples where numbers as a criteria failed or made the diagnosis impossible.
\end{abstract}

Keywords: numbers; radiology; reference value; personalized medicine; criteria

\section{Commentary}

A case of a $47-$ old female with facial and neck pain which fits with symptoms of eagle syndrome. The length of the elongated styloid processes were and measured $3.1 \mathrm{~cm}$ and $3.15 \mathrm{~cm}$ but both measurement are below the number criteria which are $3.2 \mathrm{~cm}$. The elongated styloid processes and ossified stylo-hyoid ligaments on both sides cause compression on facial and neck nerves which result in severe pain.

Another example is herniated cerebellum or cerebellar tensile with or without brainstem involvement which divided into benign tonsillar ectopia or low-lying tonsils $(<5 \mathrm{~mm})$, cerebellar tonsillar ectopia ( $>5 \mathrm{~mm}$ ) tonsils only herniated, Chiari malformation I ( $>5 \mathrm{~mm})$, Chiari malformation 1.5 (>12 mm) tonsils and brainstem herniated, etc. These numbers for all symptomatic or asymptomatic patients. The numbers used in the criteria are not useful with many patients. Similar to anatomical variations there are reference values variations as well.

Any cerebral calcification must be above $300 \mathrm{HU}$ to be considered a calcification, but there are countless patient with dural calcifications that scores below $300 \mathrm{HU}$, so the number criteria is not practical again. Another example is the exposure factors which vary from an obese patient to a slim patient, an adult to a child, a pregnant (who have a medical necessity) to a non-pregnant women. Even in the same category for example a neonate need a different exposure factors compared to a one-year old baby and both are in the pediatric category. Another example, the amount of intravenous (IV) contrast media which has a specific rule which is a 1 and $1 / 2$ millimeter for each kilogram which sometime is not low contrast media in many cases based on clinical experience. Another example is radiation dose where a some radiation does might cause too much harm to a particular individual and make no harm to another individual.

All the previous examples prove one point that personalized medicine must be used more in radiology. Not all the standard numbers and reference values are carved in stone and can't be changed. Plus, every person is different and these numbers are used to help us, but they can be neglected when it becomes a contradictory and limitation factor. 